\title{
Evaluation of Anti-Retroviral Drugs Effects on Liver Function Tests of HIV Infected Individuals
}

\author{
Olayonwa Damilare Ezekiel ${ }^{1}$ (D) $\triangle$ and Oyedeji Samuel ${ }^{2}$ (ID) \\ ${ }^{12}$ Department of Medical Laboratory Science, Achievers University Owo, Nigeria \\ $\triangle$ Corresponding Author: Olayonwa Damilare Ezekiel, E-mail: damilareolayonwa@gmail.com
}

\author{
ARTICLE INFORMATION \\ Received: July 08, 2021 \\ Accepted: August 25, 2021 \\ Volume: 2 \\ Issue: 2 \\ DOI: $10.32996 / j m h s .2021 .2 .2 .4$
}

\section{KEYWORDS}

Antiretroviral, alkaline

phosphatase, alanine

aminotransferase, aspartate

aminotransferase, bilirubin total.

\section{ABSTRACT}

The Human Immunodeficiency Virus (HIV) continues to be a major global public health issue, having claimed more than 35 million lives so far. Antiretroviral therapy, the drug used to treat HIV patients, had been reported to have an adverse effect on patients' livers. Therefore, this research aims to assess the parameters for measuring liver injury of HIV patients undergoing antiretroviral therapy in Owo and to determine the patients' vulnerability to liver injury. The study sample was divided into five groups comprising Control groups and groups with 6 months, 1 year, 3 years and 5 years' periods of administration of an antiretroviral drug. Serum was separated from their blood and values of ALT, ALP and BILT were determined. The results of profiling the patients based on values ALT, ALP and BILT indicated that $73 \%, 71 \%$ and $59 \%$ of the patients are within the reference range of the parameters, respectively. Further analysis of the percentage of patients likely to have liver diseases indicated that only $3.2 \%$ are prone to liver injury. The results of the One-way Analysis of Variance of the mean values of the groups on ALT, ALP and BILT indicated differences in mean values of the groups. It is suggested that a longitudinal study should be carried out to determine the effect of seasonal variation in the value of the studied parameters. It is also suggested that a wider interval of the period for the groups should be used in the future to determine whether there will be a relationship between the period of administration of the drug and the parameters.

\section{Introduction}

The Human Immunodeficiency Virus (HIV) continues to be a major global public health issue, having claimed more than 35 million lives so far. In 2017, 940000 people died from HIV-related causes globally. Approximately 36.9 million people were living with HIV at the end of 2017 with 1.8 million people becoming newly infected in 2017 globally. African Region is the most affected region, with 25.7 million people living with HIV in 2017. The African region also accounts for over two-thirds of the global total of new HIV infections (WHO, 2018). In 2016, Nigeria had 220000 new HIV infections and 160 000 AIDS-related deaths (UNAIDS, 2018). In 2017 in Nigeria, there were 3,100,000 HIV cases and 150,000 recorded deaths from HIV/AIDS (WHO, 2018).

Antiretroviral therapy is a significant treatment and control of HIV globally. In 2017, 21.7million people were accessing antiretroviral therapy. Also, 59\% of adults aged 15years and older living with HIV had access to treatment, as did $52 \%$ of children aged $0-14$ years. While $80 \%$ of pregnant women living with HIV had access to antiretroviral medicines to prevent transmission of HIV to their babies in 2017 (UNAIDS, 2018).

However, antiretroviral therapy on HIV patients has been reported to have adverse effects on patients' livers. About $8 \%$ to $23 \%$ of HIV-infected patients receiving highly active antiretroviral treatment (HAART) develop liver damage (Yimer et al., 2014).

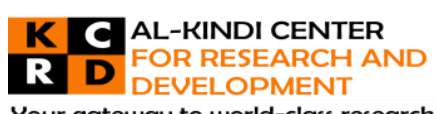

Your gateway to world-class research

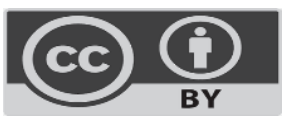

Published by Al-Kindi Center for Research and Development, United Kingdom. Copyright (c) the author(s). This open access article is distributed under a Creative Commons Attribution (CC-BY) 4.0 license 
Because the liver is responsible for the accumulation and metabolism of the majority of medications, it is a prime target for medication-induced damage (David \& Hamilto, 2010). Therefore, intake of some drugs can cause direct injury to the liver while the liver can transform others into chemicals that can cause injury to the liver directly or indirectly. The drug-induced damages to the liver are normally categorised into Herb induced liver injury (HILI) and drug-induced liver injury (DILI) (Amadi \& Oriaskwe, 2018). These damages to the liver are caused by physician-prescribed medications, over-the-counter medications, vitamins, hormones, herbs, drugs, and environmental toxins.

The literature revealed that the type and incidence of DILI display wide differences between population and geographical location (Yimer et al., 2014). There was no reported study of incidence of DILI of HIV patients on antiretroviral therapy in Owo. As HIV patients in Owo are of different populations and in different geographical locations, there is a need to investigate the incidence of DILI of antiretroviral therapy on the population to contribute to existing literature.

Analysis of liver injury associated with antiretroviral therapy is hampered because toxicity has not been reported uniformly. The available information is quite heterogeneous: case reports or studies on small numbers of patients mainly report fatal or nearfatal side effects (Spengler, Lichterfeld, \& Rockstroh, 2002). Therefore, this research work is aimed at assessing the parameters of measuring liver injury of HIV patients undergoing antiretroviral therapy in Owo, Nigeria, to determine their vulnerability to liver damage in order to contribute to the body of existing literature

\section{Literature Review}

Yimer et al. (2014) evaluated the incidence, type, severity and predictors of antiretroviral and/or anti-tuberculosis drugs induced liver injury (DILI).

A total of 1,060 treatment-naive patients were prospectively enrolled into four treatment groups. The groups were divided based on HIV infected patients and TB-HIV co-infected patients and the types of drugs administered to them. The patient's liver enzymes were monitored for 24 weeks. CD4 and HIV viral load were measured in 24th and 48th weeks.

The liver test done included examination of; aspartate aminotransferase (AST), alanine aminotransferase (ALT), alkaline phosphatase (ALP), and direct and total bilirubin were done before treatment initiation. Monitoring of liver enzymes levels was performed at baseline and on the 1st, 2nd, 4th, 8th, 12th, 24th, and 48th weeks after initiation of treatment. CD4 and HIV viral load were measured at week 24 and week 48 . For the purpose of this study, censoring was done at 24 weeks, which is the time corresponding to the completion of anti-TB therapy.

They classified the levels of aspartate aminotransferase (AST), alanine aminotransferase (ALT), alkaline phosphatase (ALP), and direct and total bilirubin into four grades thus:

- Grade 1: 1.25-2.56 $\times$ ULN, or slightly elevated ALT or ALP reaching criteria for DILI and total bilirubin $\geq 26 U L N$

- Grade 2: 2.6-5.0 $\times$ ULN, or moderate elevated ALT or ALP reaching criteria for DILI and total bilirubin $\geq 26 \mathrm{ULN}$.

- Grade 3: 5.1-10 $\times$ ULN, or severe elevated ALT or ALP reaching criteria for DILI and total bilirubin $\geq 26 U L N$ and

- Grade 4: $10 \times$ ULN, or death or transplantation due to DILI

They found that a total of 159 patients (15\%) developed DILI with severity grades $1,2,3$ and 4 of $53.5 \%, 32.7 \%, 11.3 \%$ and $2.5 \%$, respectively.

Shiferaw et al.'s study (2016) determined the prevalence of liver enzyme abnormalities and identified factors associated with liver enzyme elevations among HIV-1 infected patients. 164 HAART experienced and 164 HAART naïve patients were studied. They collected blood specimens to determine alanine aminotransferase (ALT), aspartate aminotransferase (AST), CD4 count, and viral hepatitis.

They defined upper limit normal values as ALT $=34 \mathrm{IU} / \mathrm{L}$ and $\mathrm{AST}=31 \mathrm{IU} / \mathrm{L}$ for women and $\mathrm{ALT}=45 \mathrm{IU} / \mathrm{L}$ and $\mathrm{AST}=35 \mathrm{IU} / \mathrm{L}$ for men. Also, liver enzyme abnormalities were graded as follows:

- Grade 1: 1.25-2.5X the upper limit of normal (ULN)

- Grade 2: 2.6-5XULN

- Grade 3: 5.1-10XULN

- $\quad$ Grade 4: >10XULN

They found that the prevalence of liver enzyme abnormality was $20.1 \%$ and $22.0 \%$ among HAART experienced and HAART naïve patients, respectively. The HAART experienced patients had higher mean ALT than HAART naive patients. They concluded that liver enzyme abnormalities were high in both HAART experienced and HAART naïve HIV-1 infected patients. 
Mataranyika et al's. study (2017) determined the current patterns and grades of ALT elevation in Namibia's HIV/AIDS. The study population consisted of clinical and laboratory records of patients initiated on first-line ART. A total of 79 patient records that met the eligibility criteria were included in the study. The criteria of selection were that the patient must have been on first-line ART and have records of ALT measurements at month 3 or month 6.

They graded ALT elevations based on the WHO toxicity scale with the upper limits of normal (ULN) ALT levels, that is

- $\quad$ Normal or grade $1(<2.5 \times$ ULN);

- Mild - moderate elevation or grade $2(2.5-5 \times$ ULN);

- $\quad$ Severe elevation or grade 3 (>5 ULN)

- $\quad$ Grade 4 (>10 $\times$ ULN).

The normal limits of ALT levels were considered to be $0-40$ IU/L

They found that 79 patients at baseline, 77 (97.5\%) and 2 (2.5\%) had grade 1 and grade 2 ALT elevation, respectively. At 3 months' post ART initiation, out of the 72 (91.1\%) patients who had their ALT results recorded, 67 (93.1\%) had grade 1, 4 (5.6\%) had grade 2 and 1 (1.34\%) had grade 3 ALT elevation. At 6 months' post ART initiation, out of the 66 (83.5\%) patients who had their ALT determined, 62 (93.9\%) had grade 1 and 4 (6.1\%) had grade 2 ALT elevation.

They concluded that first-line ART commonly induces mild self-limiting liver enzyme elevation in Namibian HIV patients, especially in the first 3 months. Consequently, there is a need to monitor ALT levels for at least 3 months after initiation, mainly in high-risk patients, to reduce side-effect concerns.

\subsection{Methodology \\ 3.1 Sample Population}

The sample population of this study comprised the HIV patients taking antiretroviral therapy in Federal Medical Centre Owo, Nigeria. The targeted HIV patients' population were those that do not have hepatitis or tuberculosis infection since hepatitis infection causes liver damage. The population were grouped into five categories thus: the first group were controlled group that is not having HIV, the second group were those that have been receiving treatment for up to six months, the third group were those that have been receiving treatment for up to a year, the fourth group were those that have been receiving treatment for up to 3 years and the fifth group were those that have been receiving treatment up to 5 years and above.

\subsection{Sampling Method and Sample Size}

The sample frame comprised the list of HIV patients in records of Federal Medical Centre Owo. A stratified sampling method was used to select the study population from hospital records. The population were stratified based on the period they have received treatment, as in section 3.1. 31 patients were in a group of 1 year, 32 patients were in a group of 3 years, 33 patients were in group 5 years, 32 patients were in a group of 6 months and 30 normal patients were in control group so as to meet the statistical requirements of a number of samples for running parametric statistics which was adopted in this study. Therefore, a total of 158 study samples were used for the analysis. About $10 \mathrm{ml}$ of venous blood was collected from patients that have no hepatitis infection.

\subsection{Laboratory Diagnoses of Collected Samples}

The subjects' blood was first tested for hepatitis to separate the samples of those infected by the virus from a non-infected subject. Serum was separated from Blood samples collected from the patients. COBAS C111 System at Medical Laboratory Unit of Adeoyo Maternity and Teaching Hospital, Yemetu Ibadan was used to run the samples to determine the values of the parameters. The procedure used in determining the values of the parameters (ALT, ALP and BILT) involves the following steps:

1. Sample identification numbers were typed into the machine, and the continue button was pressed,

2. The test type to run was selected from the test selection button.

3. The sample tubes were placed on the instrument while the sample LED was green.

4. The samples were processed and the results were displayed on the screen.

5. The results were copied from the displayed screen.

6. The sample tubes were removed from the instrument. 


\subsection{Data Analysis}

Statistical analysis was done using the Microsoft Excel statistical package. Descriptive statistics was used to present ALT, ALP, and total Bilirubin values in the form of tables and charts. Hy's law reference range was used to identify patients that are likely to be prone to liver damage. One-Way analysis of variance was used to test whether there are statistical significant differences in the mean values of parameters used to measure liver injury in the 5 groups of HIV patients taking antiretroviral drugs. T-test statistics was used to test the differences between mean values of the parameters for the Control group and each of the remaining four groups. Regression analysis was also used to test whether there is a relationship between the period of administration of antiretroviral drugs and the values of parameters used to measure liver injury in HIV patients taking antiretroviral drugs. The dependent variable is the administration period, while independent variables are values of ALT, ALP and total Bilirubin.

\subsection{Results}

\subsection{Profile of HIV Patients Taking Antiretroviral Drug Based on Values of ALT}

The estimated values ALT of patients taking Antiretroviral Drugs in this study are categorized into three groups on the basis of those patients with values within the reference range $(10-40 \mathrm{U} / \mathrm{L})$, those with values less than a minimum value of reference range $(<10 \mathrm{U} / \mathrm{L})$ and those with values greater than a maximum value of reference range $(>40 \mathrm{U} / \mathrm{L})$.

\section{Table 1: ALT Profile of HIV Patients Taking Antiretroviral Drug}

\begin{tabular}{|l|l|l|l|l|l|l|}
\hline Value Range & 6 Months & 1 Year & 3 Years & 5 Years & Control & Grand Total \\
\hline $10-40 \mathrm{U} / \mathrm{L}$ & $32(100 \%)$ & $23(74 \%)$ & $19(59 \%)$ & $16(48 \%)$ & $26(87 \%)$ & $116(73 \%)$ \\
\hline$<10 \mathrm{U} / \mathrm{L}$ & $0(0 \%)$ & $8(26 \%)$ & $13(41 \%)$ & $17(52 \%)$ & $4(13 \%)$ & $42(27 \%)$ \\
\hline$>40 \mathrm{U} / \mathrm{L}$ & $0(0 \%)$ & $0(0 \%)$ & $0(0 \%)$ & $0(0 \%)$ & $0(0 \%)$ & $0(0 \%)$ \\
\hline Total & 32 & 31 & 32 & 33 & 30 & $158(100 \%)$ \\
\hline
\end{tabular}

Out of the total of 158 patients whose samples were analyzed for ALT, 116 of them that represented $73 \%$ have values within the normal range of $10-40 \mathrm{U} / \mathrm{L} .42$ patients representing $27 \%$ of all the patients, have ALT values less than the minimum value for the reference range while none of the patients has ALT values greater than the reference range.

\subsection{Profile of HIV Patients Taking Antiretroviral Drug Based on Values of ALP}

The estimated values ALP of patients taking Antiretroviral Drugs in this study are categorized into three groups based on those patients with values within the reference range $(30-120 \mathrm{U} / \mathrm{L})$, those with values less than the minimum value of reference range $(<30 \mathrm{U} / \mathrm{L})$ and those with values greater than the maximum value of reference range $(>120 \mathrm{U} / \mathrm{L})$ in Table 2 .

\section{Table 2: ALP Profile of HIV Patients Taking Antiretroviral Drug}

\begin{tabular}{|l|l|l|l|l|l|l|}
\hline Value Range & 6 Months & 1 Year & 3 Years & 5 Years & Control & Grand Total \\
\hline $30-120 \mathrm{U} / \mathrm{L}$ & $18(56 \%)$ & $21(68 \%)$ & $31(97 \%)$ & $24(73 \%)$ & $18(60 \%)$ & $112(71 \%)$ \\
\hline$<30 \mathrm{U} / \mathrm{L}$ & $0(0 \%)$ & $4(13 \%)$ & $1(3 \%)$ & $2(6 \%)$ & $0(0 \%)$ & $7(4 \%)$ \\
\hline$>120 \mathrm{U} / \mathrm{L}$ & $14(44 \%)$ & $6(19 \%)$ & $0(0 \%)$ & $7(21 \%)$ & $12(40 \%)$ & $39(25 \%)$ \\
\hline Total & 32 & 31 & 32 & 33 & 30 & $158(100 \%)$ \\
\hline
\end{tabular}

Out of the total of 158 patients whose samples were analyzed for ALP, 112 of them that represented $71 \%$ have values within the normal range of $30-120 \mathrm{U} / \mathrm{L}$. 7 patients that represented $4 \%$ of all the patients have ALP values less than the minimum value for the reference range while 39 patients of them that represented $25 \%$ of the patients has ALP value greater than the reference range.

\subsection{Profile of HIV Patients Taking Antiretroviral Drug Based Values of BILT}

The estimated values BILT of patients taking Antiretroviral Drugs in this study are categorized into three groups on the basis of those patients with values within the reference range $(05-21 \mu \mathrm{mol} / \mathrm{L})$, those with values less than the minimum value of reference range $(<05 \mu \mathrm{mol} / \mathrm{L})$ and those with values greater than the maximum value of reference range $(>21 \mu \mathrm{mol} / \mathrm{L})$ in $\mathrm{Table}$ 3. 
Table 3: BILT Profile of HIV Patients Taking Antiretroviral Drug

\begin{tabular}{|l|l|l|l|l|l|l|}
\hline Value Range & 6 Months & 1 Year & 3 Years & 5 Years & Control & Grand Total \\
\hline $05-21 \mu \mathrm{mol} / \mathrm{L}$ & $19(59 \%)$ & $19(61 \%)$ & $16(50 \%)$ & $11(33 \%)$ & $28(93 \%)$ & $93(59 \%)$ \\
\hline$<05 \mu \mathrm{mol} / \mathrm{L}$ & $13(41 \%)$ & $12(39 \%)$ & $13(41 \%)$ & $19(58 \%)$ & $2(7 \%)$ & $59(37 \%)$ \\
\hline$>21 \mu \mathrm{mol} / \mathrm{L}$ & $0(0 \%)$ & $0(0 \%)$ & $3(9 \%)$ & $3(9 \%)$ & $0(0 \%)$ & $6(4 \%)$ \\
\hline Total & 32 & 31 & 32 & 33 & 30 & $158(100 \%)$ \\
\hline
\end{tabular}

Out of the total of 158 patients whose samples were analyzed for BILT 93 of them that represented $59 \%$ have values within the normal range $05-21 \mu \mathrm{mol} / \mathrm{L}$. 59 patients representing $37 \%$ of all the patients, have BILT values less than minimum value for the reference range, while 6 patients representing $4 \%$ of the patients have BILT values greater than the reference range.

\subsection{Result of Analysis of Patients that are Prone to Liver Damage Based on Hy's law Reference Range}

To classify HIV patients that are prone to liver injury based on Hy's law reference range, both ALT values must be greater than $3 \mathrm{x}$ ULN (Upper Limit of Normal) and BILT values must be greater than $3 \times$ ULN. Or both ALP values must be greater than $1.5 \times$ ULN and BILT must be greater than $3 \times$ ULN. Out of the total of 158 patients whose samples were analysed for ALP, ALT and BILT 5 of the patients represented $3.2 \%$ of all the patients who were prone to liver damage and all the 5 patients are patients with a period of administration of 1 year. 153 patients that represented $96.8 \%$ are not prone to liver damage.

\subsection{Result of Analysis of Differences in Mean Values of ALT among Groups}

The hypothesis assumed that there are no differences in ALT mean values of the groups based on the administration period of the antiretroviral drug. At 5 per cent level of significance, the hypothesis should be rejected as a P-value of 0.001579 is lower than 5 percent level of significance. Therefore, it can be concluded that at least one group mean value is different from the mean values of the remaining groups.

T-test statistical analysis of the difference between the mean values of ALT between the Control Group and the rest for the groups indicated that there are no significant differences in the mean values of ALT between Control Group, 6 months, 1 year and 3 years of drug administration as $p$ statistical values are $0.550249,0.07608$ and 0.088615 respectively. The $p$ values are greater than 5 percent level of significance; therefore, the assumption of no differences in mean values is rejected for these three groups. Only the mean values of ALT of Control Group and 5 years' period of administration indicated significant differences as $p$ statistical value is 0.008449557 less than 5 percent level of significance

\subsection{Result of Analysis of Differences in Mean Values of ALP among Groups}

The hypothesis assumed that there are no differences in ALP mean values of the groups based on a period of administration of antiretroviral drugs. Therefore, it can be concluded that at least one group mean value is different from the mean values of the remaining groups. At 5 percent level of significant the hypothesis should be rejected as P-value of $1.32 \mathrm{E}-05$ is lower than 5 percent level of significance.

T-test statistical analysis of the difference between the mean values of ALP between Control Group and the rest for the groups indicated that there are no significant differences in the mean values of ALP between Control Group, 6 months, 1 year and 5 years of drug administration as $p$ statistical values are $0.163864,0.159075$ and 0.1230925 respectively. The $p$ values are greater than 5 percent level of significance, the assumption of no differences in mean values is rejected for these three groups. Only the mean values of ALP for Control Group and group in 3 years' period of administration indicated significant differences as $p$ statistical value is $1.26 \mathrm{E}-11$ less than 5 percent level of significance.

\subsection{Result of Analysis of Differences in Mean Values of BILT among Groups}

The hypothesis assumed that there are no differences in BILT mean values of the groups based on period of administration of the antiretroviral drug. Therefore, it can be concluded that at least one group mean value is different from the mean values of the remaining groups. At 5 percent level of significant the hypothesis should be rejected as P-value of 0.02297 is lower than 5 percent level of significance.

T-test statistical analysis of the difference between the mean values of BILT between Control Group and the rest for the groups indicated that there are no significant differences in the mean values of BILT between Control Group, 1 year and 3 years of drug administration as $p$ statistical values are 0.262918 and 0.955573 respectively. The $p$ values are greater than 5 percent level of significance, the assumption of no differences in mean values is rejected for these three groups. The mean values of BILT for 
Control Group, 6 months and 5 years' administration period indicated significant differences as $p$ statistical values are $7.16 \mathrm{E}-08$ and 0.002102127 less than 5 percent level of significance.

\subsection{The Results of Analysis of Relationship between the Periods of Administration of Antiretroviral Drug and the Values of Parameters}

Regression analysis was used to explore this relationship by using period administration of the antiretroviral drug as a dependent variable and values of ALT, ALP and BILT as independent variables.

The result of the test of relationship indicated no relationship between the period of administration of antiretroviral drug and values of ALT, ALP, and BILT.

The regression coefficients for ALT are R Square 0.072754 and Adjusted R Square 0.065336 . The regression coefficients for ALP are R Square 0.041946 and Adjusted R Square 0.034281 while for BILT are R Square 0.006968 and Adjusted R Square -0.00098 . All the coefficients are less than 50 percent, so there is no indication of a relationship between the administration periods of antiretroviral drugs and ALP, ALT, and BILT values.

\section{Discussion}

This section presents the discussion of the results presented in section four. The results of profiling the patients based on values ALT, ALP and BILT indicated that $73 \%, 71 \%$ and $59 \%$ of the patients are within the reference range of the parameters, respectively. Further analysis of the percentage of patients likely to have liver diseases indicated that only $3.2 \%$ are prone to liver injury. The implication of this result is that majority of the patients are not likely to develop liver damage. This result has a lower percentage of patients likely to develop liver damage than the reported $8 \%$ to $23 \%$ by Yimer et al. (2014) and $18 \%$ by David $\&$ Hamilto (2010). The reasons for this lower percentage of patients likely to be prone to liver damage due to administration of antiretroviral might be because the patients with hepatitis B or C were screened out to exclude them from this study. Another reason might be the period when the samples were collected, as it was reported by Miyake et al. (2009) that the values of the parameters are normally influenced by the season of sample collection. They reported that the serum levels of test results tended to increase in the winter than summer and that these seasonal variations should therefore be considered when establishing either reference intervals or cut-off values.

The result of the One-way Analysis of Variance of the mean values of the groups on ALT, ALP and BILT indicated differences in means among the groups. T-test analysis of differences in mean values ALT of the Control group and each of the rest four groups indicated that there is no significant differences between ALT mean values of Control Group, groups in 6 months, 1 year and 3 years of drug administration. This finding implies no effect of an antiretroviral drug on groups in 6 months, 1 year and 3 years of drug administrations as their mean values are the same as that of the Control group. T-test of mean values patients in Control group and group in 5years period of drug administration indicated significant differences in their mean values of ALT. This finding implies that antiretroviral drug has an effect on 5 years' group as against control group. The mean values of ALT from 6 months' period of administration started decreasing up to 5 years of administration as shown in Figure 4.1. This finding implies that there is a likelihood of reduction in patients' ALT values as the period of administration increases.

T-test analysis of differences in mean values of ALP between the Control group and each of the other four groups indicated that there are no significant differences in the mean values of ALP between Control Group, groups in 6 months, 1 year and 5 years of drug administration. This finding implies no effect of antiretroviral drug on these three groups since their mean values are the same as that of the control group. Patients in the Control group and group in 3 years of administration indicated significant differences in their mean values of ALP. The implication of this finding is that antiretroviral drugs affect 3 years' period administration as their mean value is different from that of the control group.

T-test Analysis of differences in mean values of BILT between the Control group and each of the other four groups indicated that there are no significant differences in the mean values of BILT between Control Group, 1 year and 3 years of drug administration. The implication of this finding is that there is no effect of antiretroviral drugs on these two groups since their mean values are the same as that of the control group. Patients in the control group and group in 6 months and 5 years of administration indicated significant differences in their mean values of BILT. This finding implies that antiretroviral drugs affect 6 months and 5 years of period administration as their mean value is different from that of the control group.

Exploration of the relationship between periods of administration of an antiretroviral drug and the values of ALT, ALP and BILT indicated that there is no relationship between the periods of drug administration and values of the parameters. The implication of this finding is that the period of administration of antiretroviral does not have a relationship with ALT, ALP, and BILT values.

In conclusion, it is suggested that a longitudinal study should be carried out to determine the effect of seasonal variation in the value of the studied parameters. It is also suggested that a wider interval of the period for the groups should be used in the future to determine whether there will be a relationship between the period of administration of the drug and the parameters. 
Funding: This research received no external funding

Acknowledgements: I am grateful to the Management and staff of HART to HART of Federal Medical Centre Owo for allowing me to collect blood samples from HIV patients for the purpose of this project. I acknowledge the contribution of the staff of the Medical Laboratory Unit of Adeoyo Maternity and Teaching Hospital, Yemetu Ibadan, for allowing me to run my samples in their laboratory.

Conflicts of Interest: The authors declare that they have no conflict of interest.

\section{References}

[1] Ahn, J. (2011). Assessment of Liver Function and Diagnosis. Retrieved January 29, 2019, from http://www.meddean.luc.edu/: http://www.meddean.luc.edu/Lumen/meded/Medicine/medclerk/2004_05/level1/Liver/presentation_12.pdf

[2] Amadi, C. N., \& Oriaskwe, O. E. (2018, June 24). Herb-Induced Liver Injuries in Developing Nations: An Update. Retrieved January 29, 2018, from Toxic MPDI: https://www.ncbi.nlm.nih.gov/pmc/articles/PMC6027193/

[3] Ananya, M. (2018). AIDS Pathophysiology. Retrieved February 23, 2019, from News-Medical Life Sciences: https://www.newsmedical.net/health/AIDS-Pathophysiology.aspx

[4] Cachay, E. R. (2019). Human Immunodeficiency Virus (HIV) Infection. Retrieved February 23, 2019, from MSD Manual professional version: https://www.msdmanuals.com/professional/infectious-diseases/human-immunodeficiency-virus-hiv/human-immunodeficiency-virus-hivinfection

[5] David, S., \& Hamilto, P. J. (2010, January 1). Drug-induced Liver Injury. Retrieved January 29, 2019, from NCBI Resources: https://www.ncbi.nlm.nih.gov/pmc/articles/PMC3160634/

[6] Herrine, S. K. (2018). Liver Injury Caused by Drugs. Retrieved Feburary 23, 2019, from MSD Manuals Professional version: https://www.msdmanuals.com/professional/hepatic-and-biliary-disorders/drugs-and-the-liver/liver-injury-caused-by-drugs

[7] Lucien, K., Clement, A., Fon, N., Weledji, P., \& Ndikvu, C. (2010, September). The effects of antiretroviral treatment on liver function enzymes among HIV-infected out patients attending the Central Hospital of Yaounde, Cameroon. African Journal of Clinical and Experimental Microbiology, 17(3), 174-178.

[8] Luo, K. E. (2018, March 2). What does Liver do? Retrieved February 22, 2019, from Medical News Today: https://www.medicalnewstoday.com/articles/305075.php

[9] Marsden, M. D. (2017). HIV Structure, Life cycle and Replication . Retrieved February 23, 2019, from Tibotec Pharmaceuticals Ltd: http://preprod.www.tibotec.com/content/backgrounders/www.tibotec.com/hiv lifecycle.html

[10] Miyake, N., Miyake, K., Kondo, S., Tabe, Y., Ohsaka, A., \& Minda, S. (2009). Seasonal variation in liver function tests: a time-series analysis. Annals of Clinical Biochemistry, 46, 377-384.

[11] Nunez, M. (2006). Hepatotoxicity of antiretrovirals: Incidence, mechanisms and management. Journal of Hepatology, $44,132-139$.

[12] Singh, A., Bhat, T. K., \& Sharma, P. O. (2011). Clinical Biochemistry of Hepatotoxicity. Journal of Clinical Toxicology, 1-19.

[13] Spengler, U., Lichterfeld, M., \& Rockstroh, J. K. (2002). Antiretroviral drug toxicity - a challenge for the hepatologist? Journal of Hepatology, $36,283-294$.

[14] Sulkowski, M. S. (2004, March 1). Drug-Induced Liver Injury Associatedwith Antiretroviral Therapy that IncludesHIV-1 Protease Inhibitors. Clinical Infectious Diseases, 38(1), S90-S97.

[15] UNAIDS. (2018, December 1). Fact Sheet World Aids Day 2018. Retrieved February 22, 2019, from 2017 GLOBAL HIV STATISTIC: http://www.unaids.org/sites/default/files/media_asset/UNAIDS_FactSheet_en.pdf

[16] Wambani, J. R., Ogola, P. E., Arika, W. M., Rachuonyo, H. O., Kemboi, N. G., \& Lihana, R. (2015). Anti-Retroviral Drug Hepatotoxicity and Risk Factors in HIV Patients with or Without Hepatitis B and C: A Review. Journal of Infectious Diseases and Therapy, 3(6), 1-5.

[17] WHO. (2018). HIV/AIDS. Retrieved February 22, 2019, from Global Health Observatory (GHO) data: https://www.who.int/gho/hiv/en/

[18] WHO. (2018, July 11). Number of deaths due to HIV/AIDS. Retrieved February 23, 2019, from Global Health Observatory data repository: http://apps.who.int/gho/data/node.main.623?lang=en

[19] Yimer, G., Gry, M., Amogne, W., Makonnen, E., Habtewold, A., \& Petros, Z., (2014, April). Evaluation of Patterns of Liver Toxicity in Patients onAntiretroviral and Anti-Tuberculosis Drugs: AProspective Four Arm Observational Study in Ethiopian Patients. Plosone Journal, 9(4), 1-10. 